\title{
Associations Between Inflammation and Cognitive Function in African Americans and European Americans
}

\author{
B. Gwen Windham, MD, MHS, ${ }^{* 1}$ Brittany N. Simpson, BA, ${ }^{\dagger 1}$ Seth Lirette, MS, ${ }^{*}$ John Bridges, MS, ${ }^{\dagger}$ \\ Lawrence Bielak, DDS, MPH, ${ }^{\S}$ Patricia A. Peyser, PhD ${ }^{\S}$ Iftikhar Kullo, MD, "Stephen Turner, MD," \\ Michael E. Griswold, PhD, ${ }^{*}$ and Thomas H. Mosley, PhD*
}

OBJECTIVES: To examine associations between specific inflammatory biomarkers and cognitive function in African Americans (AAs) and European Americans (EAs) with prevalent vascular risk factors.

DESIGN: Cross-sectional analysis using generalized estimating equations to account for familial clustering; standardized $\beta$-coefficients, adjusted for age, sex, and education are reported.

SETTING: Community cohort study in Jackson, Mississippi, and Rochester, Minnesota.

PARTICIPANTS: Genetic Epidemiology Network of Arteriopathy (GENOA)-Genetics of Microangiopathic Brain Injury (GMBI) Study participants.

MEASUREMENTS: Associations between inflammation (high-sensitivity C-reactive protein (CRP), interleukin (IL)-6, soluble tumor necrosis factor (TNF) receptor 1 and 2 (sTNFR1, sTNFR2)) and cognitive function (global, processing speed, language, memory, and executive function) were examined in AAs and EAs $(\mathrm{N}=1,965$; aged 26-95, 64\% women, 52\% AA, 75\% with hypertension).

RESULTS: In AAs, higher sTNFR2 was associated with poorer cognition in all domains (global: $-0.11, P=.009$; processing speed: $-0.11, P<.001$; language: -0.08 , $P=.002$; memory: $-0.09, P=.008$; executive function: $-0.07, P=.03)$; sTNFR1 was associated with slower processing speed $(-0.08, P<.001)$ and poorer executive function $(-0.08, P=.008)$; higher $\mathrm{CRP}$ was associated with slower processing speed $(-0.04, P=.024)$, and higher IL6 was associated with poorer executive function $(-0.07$, $P=.02$ ). In EA, only higher sTNFR1 was associated with

From the "Division of Geriatric Medicine, Department of Medicine, University of Mississippi Medical Center; ${ }^{\dagger}$ School of Medicine, University of Mississippi Medical Center; ${ }^{\star}$ Center of Biostatistics and Bioinformatics, University of Mississippi Medical Center, Jackson, Mississippi; ${ }^{\S}$ School of Public Health, University of Michigan, Ann Arbor, Michigan; and "Mayo Clinic, Rochester, Minnesota.

${ }^{1}$ These authors contributed equally to this work.

Address correspondence to B. Gwen Windham, 2500 N. State Street; Jackson, MS 39216. E-mail: gwindham@umc.edu

DOI: $10.1111 /$ jgs. 13165 slower processing speed $(-0.05, P=.007)$. Associations were not found between cognition and sTNFR2, CRP, or IL6 in EA.

CONCLUSION: In a population with high vascular risk, adverse associations between inflammation and cognitive function were especially apparent in AAs, primarily involving markers of TNF $\alpha$ activity. J Am Geriatr Soc 62:23032310, 2014.

Key words: inflammation; cognition; ethnicity

$\mathrm{D}$ ementia affects approximately 5 million people in the United States, with Alzheimer's disease (AD) accounting for $60 \%$ to $80 \%$ of these cases and vascular cognitive impairment accounting for most of the remainder. Vascular disease causes cognitive impairment and amplifies the deleterious effects of $\mathrm{AD}$ pathology by lowering the threshold for cognitive impairment and augmenting the trajectory of cognitive decline. ${ }^{1-3}$ Growing evidence suggests that inflammation may contribute to the pathophysiology of $\mathrm{AD}$ and vascular dementia $(\mathrm{VaD}) .{ }^{4}$ Furthermore, studies demonstrating that adding inflammatory markers to the model improves predictive ability of lipid markers in cardiovascular disease outcomes ${ }^{5}$ provide face validity for an inflammatory-mediated role in vascular disease of the brain, similar to that of other end organs.

Associations between cognitive function and interleukin-6 (IL6), ${ }^{6-9}$ tumor necrosis factor (TNF)- $\alpha$ and soluble TNF receptors (sTNFRs), ${ }^{10-12}$ and C-reactive protein $(\mathrm{CRP})^{8,}{ }^{3-17}$ have been reported. Inflammation may be involved differently in $\mathrm{VaD}$ and $\mathrm{AD}$ and in different racial and ethnic groups. For example, TNF $\alpha,{ }^{18} \mathrm{CRP}$, and IL $6{ }^{15}$ are higher in persons with $\mathrm{VaD}$ than in those with $\mathrm{AD}$ and may be important risk factors for cognitive impairment in persons with cardiovascular risk factors. Although African Americans (AAs) may be more likely to have dementia than European Americans (EAs), ${ }^{19}$ few studies of inflammation and cognitive function have included AAs. ${ }^{6,7,13}$ Inflammatory biomarker levels appear to differ between AAs and EAs ${ }^{20-22}$ 
and AAs may have stronger responses to inflammatory stimuli than EAs. ${ }^{23}$ In addition, some $\mathrm{e}^{20,21,24,25}$ but not all studies $^{26}$ suggest that levels and actions of inflammatory markers differ in EAs and AAs and may contribute differently to the pathophysiology of dementia. The purpose of this study was to examine associations between CRP, IL6, and TNF $\alpha$ activity and cognitive function in EAs and AAs with prevalent cardiovascular risk factors, all of whom had hypertension or two siblings with hypertension before age 60 .

\section{METHODS}

\section{Population}

The Genetic Network of Arteriopathy (GENOA) study, begun in 1995, follows a well-characterized cohort of individuals with hypertension and their siblings recruited from Jackson, Mississippi (AA only) and Rochester, Minnesota (EA only) ( $\mathrm{N}=3,437 ; 66 \%$ female, 57\% AA, aged 28-91, $52 \%$ obese at baseline). At least two members of each sibship had hypertension before age 60 at enrollment. Inflammatory markers were assayed at the second examination (GENOA Visit 2, 2000-04). Neurocognitive testing was conducted at or after Visit 2 (The Genetics of Microangiopathic Brain Injury [GMBI], 2001-06; hereafter included with Visit 2). Participants in Visit 2 and GMBI $(\mathrm{n}=2,721)$ included 1,239 individual EAs (469 full-sibling pairs) and 1,482 individual AAs (626 full-sibling pairs). Of these, 162 (3 EA, $159 \mathrm{AA}$ ) were missing information on all inflammatory markers, and 10 self-reported a history of dementia and were excluded, leaving 2,549 participants. Cognitive data were available for 1,965 (960 EA, 1,005 AA), who constitute the analysis dataset, including those in the dataset for the sensitivity analyses, which addresses potential bias of missing data. Of these, 1,857 (95\%) had inflammatory biomarker data and constitute the completers data set.

\section{Inflammatory Markers}

At Visit 2, fasting blood samples were centrifuged for 10 minutes at $4{ }^{\circ} \mathrm{C}$, aliquoted in $0.5-$ to $1-\mathrm{mL}$ volumes of ethylenediaminetetraacetic acid plasma (serum for CRP), and stored at $-80^{\circ} \mathrm{C}$ within 2 hours of venipuncture; frozen samples were shipped to the Mayo Clinic Immunochemical Core Laboratory (Rochester, MN) overnight on dry ice. CRP assays were performed using immunoturbidometric assays (Diasorin, Inc., Stillwater, MN; interassay imprecision $1.8-2.6 \%$; intraassay imprecision $1.0-9.2 \%$ ), and multiplex assays (SearchLight, Pierce, Boston, MA) were used for IL-6 and sTNFRs; sTNFR fractions show stability over time, with longer half-lives than TNF $\alpha$ levels, and have been validated as sensitive indicators of TNF- $\alpha$ system activation. ${ }^{27,28}$ Precision of the assays performed using SearchLight was retrospectively determined based on data derived from a blinded internal plasma control sample. Algorithms were developed to reduce plate-to-plate variations in protein levels, and all analyses used these normalized data. ${ }^{29}$

\section{Cognitive Testing}

Neurocognitive tests were offered to all participants in the same sequence using standardized protocols to assess global mental status, memory, language, processing speed, and executive function. All scores were ordered so that higher values reflect better cognition, and standardized coefficients were used to allow comparisons across measures.

\section{Global Cognitive Function}

The Mini-Mental State Examination (MMSE, range 0 [worst] to 30 [best] $)^{30}$ was administered according to protocol consistent with the Consortium for the Establishment of a Registry for Alzheimer's Disease battery. ${ }^{31,32}$

\section{Processing Speed}

The Wechsler Adult Intelligence Scale Revised Digit Symbol Substitution Task was used to test complex visual attention, sustained and focused concentration, response speed, and visuomotor coordination, and the Trail-Making Test Part A (TMT-A) was used to measure visual conceptual and visuomotor tracking, attention, sequencing, mental flexibility, visual search, and motor function (nearest 0.01 seconds, maximum 4 minutes). ${ }^{32}$ Because slower times indicate poorer performance, times were multiplied by -1 for analyses so that higher numbers represented better performance.

\section{Memory}

The Rey Auditory Verbal Learning Test (range 0-15) assesses learning and memory using multiple learning trials and a 30-minute delayed recall of 15 items on a list. ${ }^{32}$ The Wechsler Adult Intelligence Scale III Incidental Learning Task allows continuation of the Digit Symbol Substitution Task until the third row of the test has been completed. ${ }^{33}$ After a 5-minute delay, the symbol pairs with free-recall ${ }^{32}$ are presented again.

\section{Language}

The FAS was used to measure letter fluency; participants must spontaneously produce words beginning with a specific letter (F, A, S) within 60 seconds. ${ }^{32}$ The Animal Naming Task was used to measure category (animals) fluency. ${ }^{31}$

\section{Executive Function}

The TMT-B was used to assess attention, sequencing, mental flexibility, visual search and motor function using time and error counts. ${ }^{32}$ Times were multiplied by -1 so that higher scores represented better function.

\section{Composite Cognitive Domain Measures}

Composite measures for processing speed, memory, and language domains were constructed from two tests within each domain to reduce measurement error and floor and ceiling effects of individual tests. ${ }^{34-36} \mathrm{~A}$ standardized $z$-score was created for each measure, and $z$-scores were averaged within a domain to create the composite. ${ }^{34-36} \mathrm{~A}$ factor-analytical combination method for constructing the domain scores yielded similar associations between 
inflammation and cognitive measures (results available on request).

\section{Covariates}

Blood pressure, measured three times in a seated, resting state with appropriately sized cuffs, was defined as the average of the second and third measurements. Hypertension was defined as measured blood pressure greater than $140 / 90$, self-report of high blood pressure, or antihypertensive medication use. Diabetes mellitus was defined as fasting glucose of $126 \mathrm{mg} / \mathrm{dL}$ or greater, random glucose of $200 \mathrm{mg} / \mathrm{dL}$ or greater, self-report of diabetes mellitus, or hypoglycemic medication use. Antianxiety, antidepressant, hypnotic, and narcotic medications and sleep aids (over the counter or prescription) taken in the previous 2 weeks were classified as medications with potential to affect cognition. Never-smoker was defined as having never smoked more than 100 cigarettes. Height was measured using a stadiometer and weight using an electronic balance with participants wearing lightweight clothes. Body mass index (BMI) was calculated as weight $(\mathrm{kg}) /$ height ${ }^{2}\left(\mathrm{~m}^{2}\right)$.

\section{Statistical Analysis}

Associations between inflammatory markers and each cognitive domain were estimated using linear models fit with generalized estimating equations (GEEs) to account for familial clustering and Huber-White robust standard error estimates. Because inflammatory markers and cognitive function scores all use different measurement units, standardized outcomes and predictors were modeled to facilitate comparison of models. Thus, a beta coefficient of -0.5 is interpreted as a 0.5 -standard deviation (SD) decrease in the cognitive score outcome being associated with a 1-SD increase in the inflammatory marker. Diagnostic lowess smoothers revealed linear relationships on the natural scale, inflammatory markers were only mildly skewed, and estimates were resistant to any extreme value effects (Figure 1), hence associations are presented with standardized, non-log-transformed inflammatory markers. Primary adjusted models included age, education, and sex and accounted for familial clustering. Extended adjusted models also included diabetes mellitus, hypertension, BMI, smoking, stroke history, alcohol, lipid-lowering medications, and central nervous system medications. Differences according to race were examined using interaction terms, acknowledging that race and site are aliased by design (meaning that all AAs are from one site and all EAs from another). Results are presented stratified according to race as shorthand for AA (MS)/EA (MN) race (site) groups.

Characteristics of participants who had and who were missing cognitive data were evaluated according to race, and sensitivity analyses were conducted using weighted GEEs to examine the robustness of findings after accounting for missing cognitive data. Analyses were performed using STATA 12 (Stata Corp., College Station, TX).
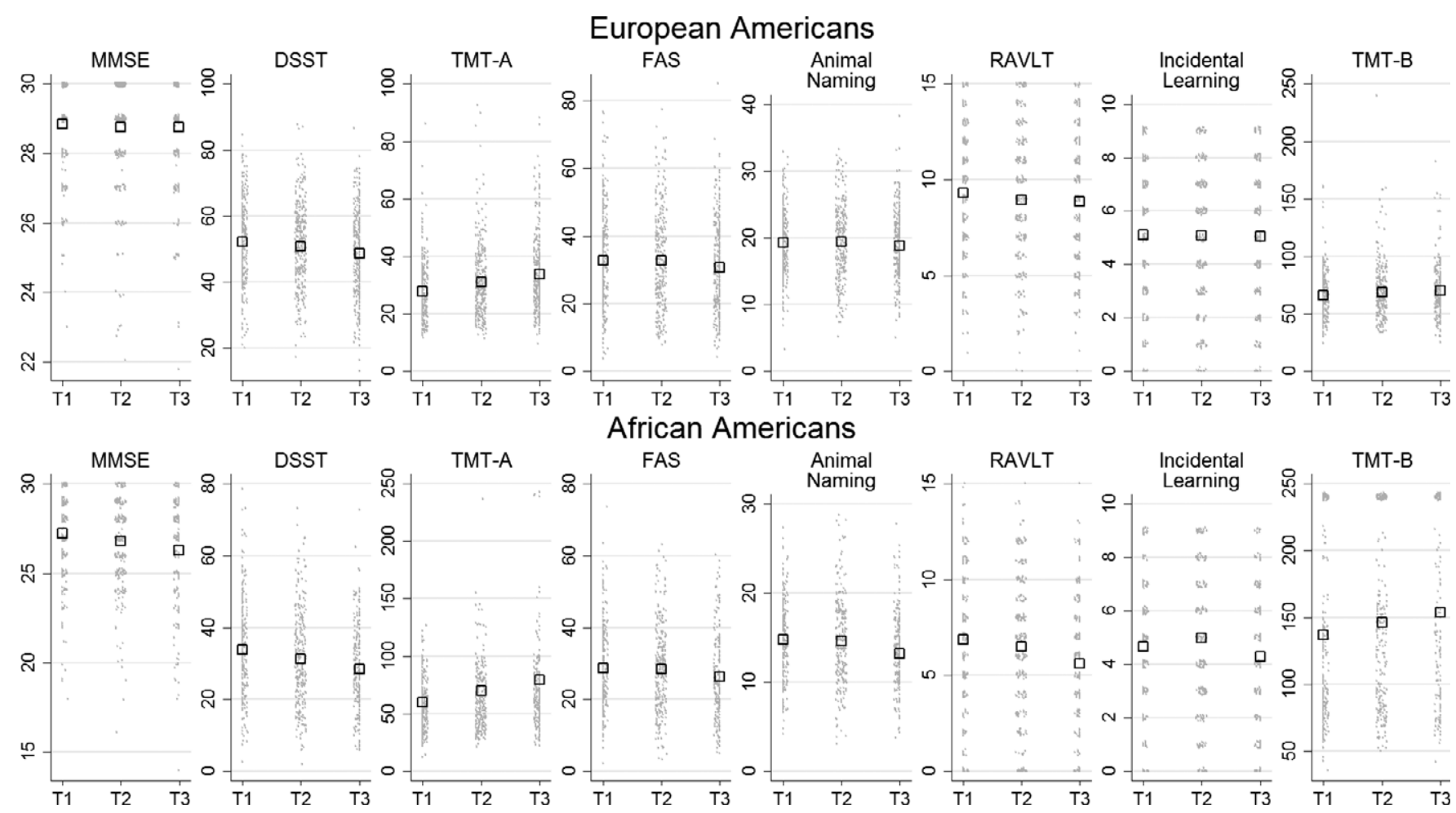

Figure 1. Raw cognitive score means according to race-stratified soluble tumor necrosis factor receptor 2 inflammatory tertile. Open squares indicate raw cognitive score means with individual raw cognitive scores displayed as points within each tertile. MMSE = Mini-Mental State Examination; DSST = Digit Symbol Substitution Task; TMT-A = Trail-Making Test Part A; RAVLT = Rey Auditory Verbal Learning Test; Incidental Learning-Wechsler Adult Intelligence Scale-III Incidental Learning Task; TMT-B-Trail-Making Test Part B; T1 = Tertile 1; T2 = Tertile 2; T3 = Tertile 3. 


\section{RESULTS}

Characteristics are shown for participants with inflammatory marker data, stratified according to race and presence versus absence of cognitive data (Table 1). AAs were older and more likely to be female, to have diabetes mellitus, to have hypertension, to have ever smoked, to have a higher BMI, and to have higher CRP and IL6 and lower sTNFR1 levels; and sTNFR2 levels were similar in AAs and EAs. MMSE scores ranged from 14 to 30 (mean $27.9 \pm 2.3$ ). Fourteen AAs $(2.0 \%)$ and eight EAs $(1.1 \%)$ met racespecific criteria for cognitive impairment. ${ }^{37}$

All cognitive data were missing in $271(22 \%)$ EAs and $421(32 \%)$ AAs. In EAs, missing data were associated with being younger $(P=.02)$ and male $(P=.008)$ and having diabetes mellitus $(P=.04)$. AAs who were missing cognitive data were more likely to have diabetes mellitus $(P=.003)$ and to have a higher BMI $(P=.03)$, a lower education level $(P<.001)$, and higher levels of all inflammatory markers (sTNFR1, $P=.005$; sTNFR2, $P=.006$; CRP, $P=.01$; and IL6, $P=.006$ ) than AAs with cognitive data (Table 1).

Mean race-stratified cognitive scores according to inflammatory tertiles differed in AAs and EAs (Figure 1). For example, in AAs, all cognitive measures were worse with increasing tertile of sTNFR2. In EAs, only processing speed $(P<.001)$ and executive function $(P=.03)$ were associated with sTNFR2 (Table 2, Figure 1). In AAs, sTNFR1 was also associated with cognitive domains (all $P<.05$ ), except for memory, whereas in EAs, processing speed $(P<.001)$, language $(P=.011)$, executive function $(P<.001)$, and marginally memory $(P=.097)$ were associated with sTNFR1. CRP was associated with memory in
EAs $(P=.04)$, but no associations were observed between cognition and CRP or IL6 in AAs. IL6 was associated with processing speed $(P<.001)$, language $(P<.002)$, and executive function $(P=.03)$ in EAs (Table 2, Figure 1).

In adjusted models, every 1-SD increase in sTNFR2 was associated with a 0.11-SD lower MMSE score in AA $(\beta=-0.11,95 \%$ confidence interval $(\mathrm{CI})=-0.20$ to $0.03, P=.009)$. The data did not support a similar association in EAs $(\beta=0.03,95 \% \mathrm{CI}=-0.01-0.07, P=.14)$; the interaction term supported a different relationship according to race $(\beta=-0.14,95 \% \mathrm{CI}=-0.24$ to -0.05 , $P=.003$, Figure 2, Table 3). Similar to the MMSE outcome, the data supported inverse associations between sTNFR2 and all other cognitive domains for AAs but not EAs. (Table 3, Figure S1) Each SD increase in sTNFR2 in AAs was associated with poorer performance in processing speed $(\beta=-0.11,95 \% \mathrm{CI}=-0.16$ to $-0.07, P<.001)$, language $(\beta=-0.08, \quad 95 \% \quad C I=-0.13$ to -0.03 , $P=.002)$, memory $(\beta=-0.0,95 \% \mathrm{CI}=-0.16$ to -0.02 , $P=.008)$, and executive function $(\beta=-0.07,95 \%$ $\mathrm{CI}=-0.13$ to $-0.01, P=.03)$. The inferences were the same in extended adjusted models (data available on request).

Higher sTNFR1 in AAs was associated with slower processing speed $(\beta=-0.08,95 \% \mathrm{CI}=-0.12$ to -0.04 , $P<.001$ ) and executive function in adjusted models (Figure S1, Table 3: $\beta=-0.0895 \% \mathrm{CI}=-0.14$ to -0.02 , $P=.008)$. The associations between sTNFR1 and MMSE or language in AAs were similar in magnitude but did not reach statistical significance (MMSE: $\beta=-0.07,95 \%$ $\mathrm{CI}=-0.16-0.0, \quad P=.09 ; \quad$ language: $\quad \beta=-0.04, \quad 95 \%$ $\mathrm{CI}=-0.09-0.00, P=.07)$. In AAs, higher CRP was associated with slower processing speed $(\beta=-0.04,95 \%$

Table 1. Participant Characteristics According to Race and Cognitive Data

\section{European Americans}

\begin{tabular}{lc}
\hline With Cognitive & Missing Cognitive \\
Data, $n=960$ & Data, $n=272$
\end{tabular}

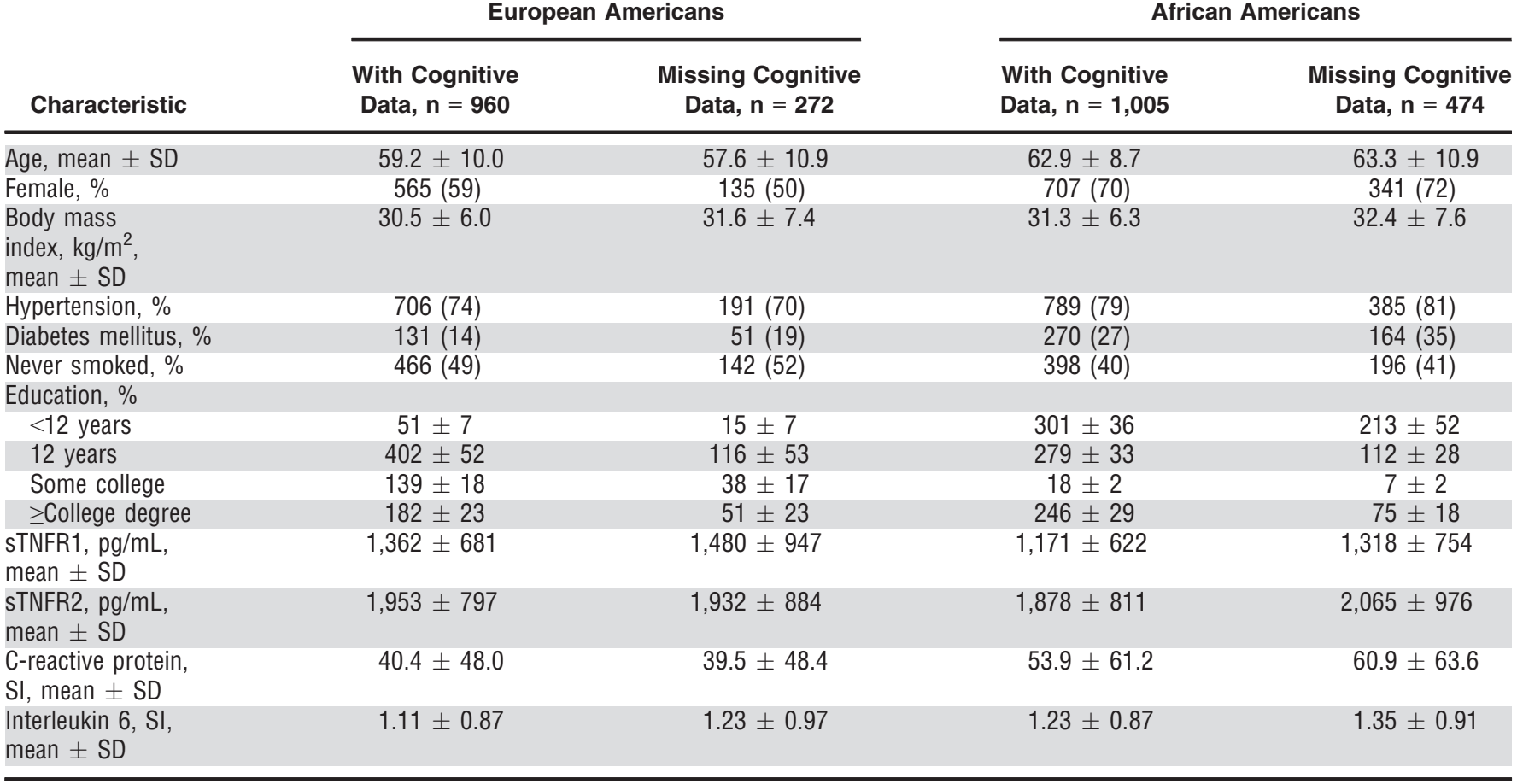

African Americans 


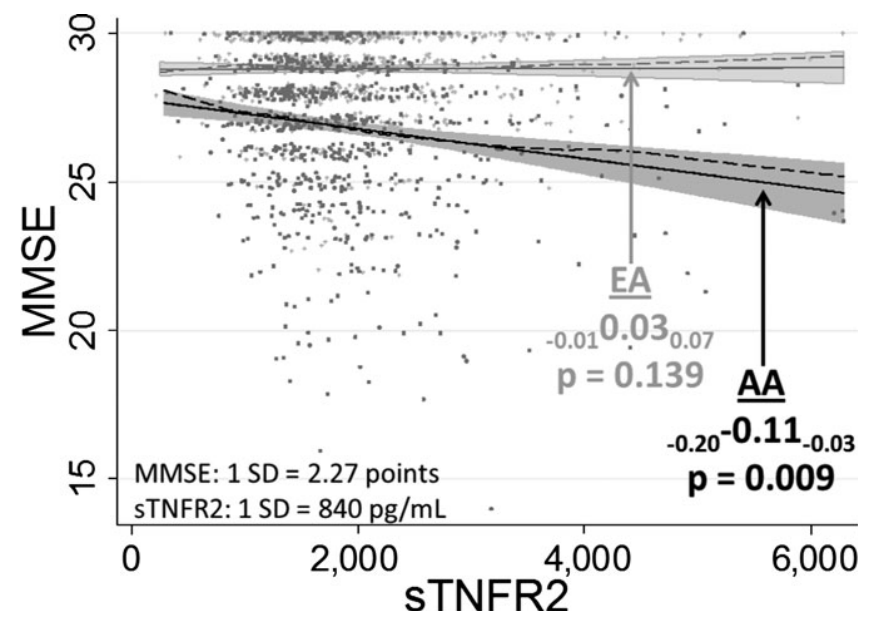

Figure 2. Differential associations between soluble tumor necrosis factor receptor 2 (sTNFR2) and Mini-Mental State Examination (MMSE) score in African Americans (AAs: dark gray) and European Americans (EAs: light gray) from adjusted models. Solid lines are regression lines with shaded confidence bounds. Dashed lines are LOWESS nonlinear smoothers (diagnostic check). Race specific standard deviations (SDs) and standardized beta coefficients are shown with subscripted lower and upper 95\% confidence limits (lower confidence limit "LCL" and upper confidence limit "UCL"), displayed as ${ }_{\mathrm{LCL}} \beta_{\mathrm{UCL}}$.

$\mathrm{CI}=-0.070$ to -0.010$) P=.02$ ), and higher IL6 was associated with poorer executive function $(\beta=-0.07,95 \%$ $\mathrm{CI}=-0.12$ to $-0.01, P=.02$ ) (Table 3, Supplemental Figure).

In EAs, sTNFR1 was associated only with processing speed $(\beta=-0.05,95 \% \mathrm{CI}=-0.08$ to $-0.01, P=.007)$; sTNFR2, IL6, and CRP were not statistically associated with any cognitive domain (Supplemental Figure, Table 3).

Sensitivity analyses using weighted GEEs suggested that the reported results comparably or more conservatively estimate associations between inflammatory markers and cognitive function (Supplementary Table).

\section{DISCUSSION}

In a cohort of EAs and AAs with hypertension and their siblings (with and without hypertension), substantial proportions of whom had other risk factors for cardiovascular disease, inflammatory markers were differently associated with cognitive function in AAs and EAs. In AAs, a biomarker of TNF $\alpha$ activity was associated with five domains of cognitive function, whereas IL6 and CRP were associated only with executive function and processing speed, respectively; in EAs, only markers of TNF $\alpha$ activity were associated with a single cognitive domain, processing speed. Although the cohort overall may be considered at high risk of cardiovascular disease, cardiovascular risk factors were also more prevalent in AAs than EAs. These findings suggest that, in these young to old AAs with hypertension or a strong family history of hypertension and prevalent cardiovascular risk factors, TNF $\alpha$ activity, and perhaps IL6 and CRP, may be important risk factors for cognitive dysfunction. 


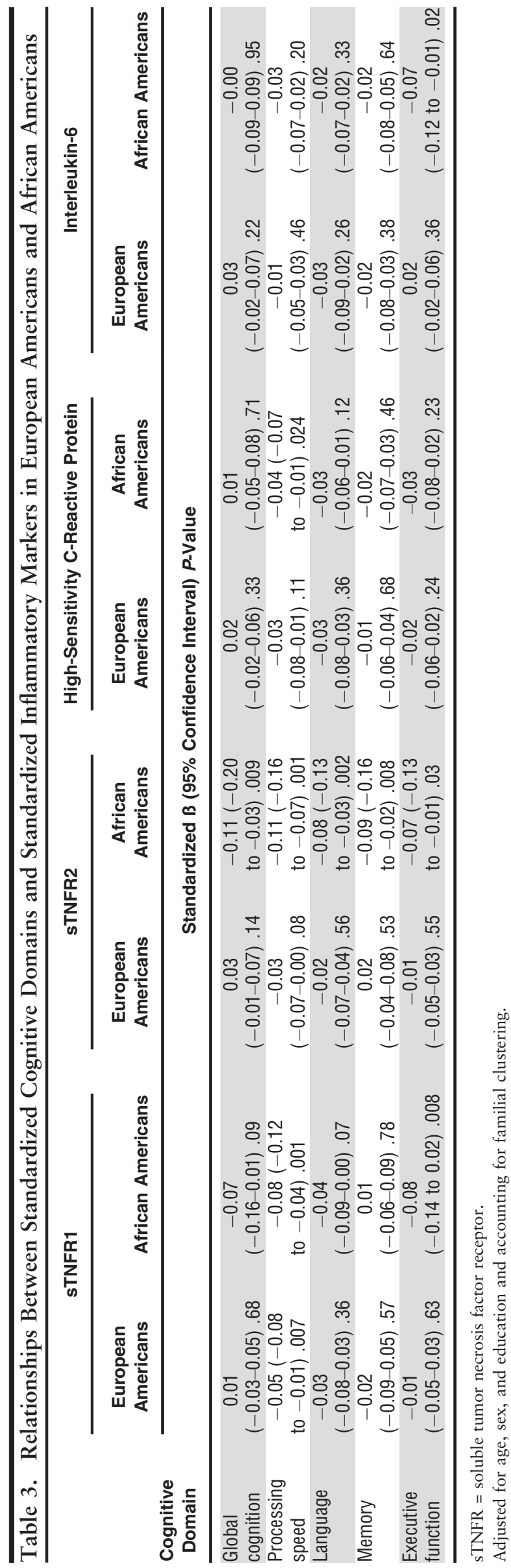

One explanation for these findings in this population could involve inflammatory-mediated cerebrovascular disease. Cardiovascular risk factors, inflammation, 5,7,13,38,39 brain structure abnormalities, ${ }^{40}$ and poor cognitive function, ${ }^{41,42}$ particularly executive function ${ }^{41}$ and processing speed, ${ }^{41}$ are interrelated. Mechanisms linking blood pressure to cognition are especially relevant for this study population and have been classified as functional (e.g., endothelial dysfunction or vascular dysregulation; altered blood flow, including nocturnal dipping patterns; reduced amyloid clearance), structural (e.g., white matter hyperintensities, atrophy), pharmacological (related to renin-angiotensin system), stroke related, and other (including hypertension with insulin resistance or impaired insulin signaling centrally). ${ }^{43}$ AAs are disproportionately burdened by cardiovascular disease ${ }^{44}$ and may exhibit heightened responses and greater endothelial dysfunction in response to inflammatory stimuli in vasculature, specifically TNF $\alpha$ pathways. ${ }^{23}$ Furthermore, TNF $\alpha$ upregulation has been observed in individuals with hypertension, ${ }^{45}$ and more than $70 \%$ of this cohort was hypertensive, whereas the remainder had at least two siblings with hypertension before age 60 . Thus, inflammation may mediate cognitive decline through arteriosclerotic disease in the brain but may also adversely affect cognition through direct effects of inflammation on synaptic plasticity, neurogenesis, and neuromodulation that affect cognition. ${ }^{46}$ High inflammatory biomarker levels can occur for a number of reasons, and mechanisms explaining the link between inflammation and cognition in this crosssectional cohort study could not be elucidated, although these findings can be considered hypothesis generating in a sample of AAs most of whom had hypertension.

These findings further complement and expand upon those of other studies linking inflammation and cognition $^{4,6-10,13-18,47}$ by reporting findings from a relatively large AA cohort across a broad age range. Studies in older adults demonstrate associations between higher TNF $\alpha$ activity and poorer cognitive function, ${ }^{10}$ higher TNF $\alpha$ activity in individuals with $\mathrm{VaD}$ than in those with $\mathrm{AD},{ }^{18}$ and higher TNF $\alpha$ activity in individuals with $\mathrm{VaD}$ or $\mathrm{AD}$ than in controls. ${ }^{18,48}$ In this cognitively unimpaired cohort aged 28 to 91, associations were found between inflammation and poor cognitive function in AAs. A longitudinal Swedish study that showed higher baseline sTNFR1 and sTNFR2 levels in persons with mild cognitive impairment who converted to dementia than in those with mild cognitive impairment who remained stable or in controls supported these findings. ${ }^{12} \mathrm{~A}$ relationship between TNF $\alpha$ and executive function decline, but not other cognitive domains, was observed in the largely EA Framingham Offspring cohort. ${ }^{10}$ Associations were observed with processing speed only in the GENOA EA. Differences in the cohorts' prevalent cardiovascular risk factors, with the GENOA cohort having a greater burden, might explain some of the inconsistency. In addition, the current analysis was cross-sectional, and the Framingham Offspring study was longitudinal.

The Framingham Offspring and GENOA cohorts were younger than many participants in studies of inflammation and cognition. Findings in these younger cohorts are of particular interest because interventions to halt or delay cognitive decline may be more effective in earlier stages. It would 
also be of interest to see whether interventions that target the conditions that cause inflammation are more successful than interventions that target existing inflammation.

Although the individual estimates of associations between standardized inflammatory biomarkers and standardized cognitive measures may appear small, going from lowest to highest sTNFR2 values, there was an average 2-point difference in MMSE score, which is clinically meaningful. To put the sTNFR2 effect further into context, an increase in $1 \mathrm{SD}$ of sTNFR2 $(\beta=-0.11)$ would be similar to a 5 -year difference in age (age $\beta=-0.02$ per year); across the range of sTNFR2 from 252 to $2,690 \mu \mathrm{g}$, this would be similar to 20 years of aging.

Some limitations warrant further discussion. The different results according to race could be due to regional differences in the AA and EA populations, because study sites were race specific. Regardless, the relationships are of interest. In addition to potential race and site effects, the higher risk profile in AAs (e.g., older age; more obesity, hypertension, diabetes mellitus) could contribute to differential findings. This explanation is of particular interest because it might identify mechanisms that are more relevant in populations with prevalent cardiovascular risk factors. In addition, this study was limited to four inflammatory markers (CRP, sTNFR1 and 2, and IL6), even though other proinflammatory markers have been found to be associated with cognition, including other ILs and serum amyloid A. ${ }^{49,50}$ Nevertheless, the biomarkers in the current study are among those with biological plausibility and some evidence in other studies, although mostly in EAs, that they are important in vascular and nonvascular cognitive impairment. Numerous triggers can cause inflammatory biomarkers to be high, and the influence of other conditions that increase inflammation could not be excluded, although the results remained significant even after accounting for several comorbidities associated with inflammation, including cardiovascular disease and diabetes mellitus. In addition to adjusting for other potential confounders in parsimonious and extended adjusted models, standard approaches of stratification were included, and sensitivity analyses were conducted for potential informative missingness effects. Although the cognitive measures may not detect early decline, significant relationships were observed in this population. Additional limitations include the cross-sectional design, which limits inferences of causality. Volunteer bias may limit generalizability to dissimilar populations, but the findings reported are among a few reporting such relationships in AAs. Longitudinal studies could address some limitations by assessing temporal associations between inflammation and cognitive decline.

\section{CONCLUSION}

Inflammation is increasingly recognized as an important contributor to numerous health outcomes. Deleterious effects on cognitive function may be especially apparent in AAs with vascular risk factors. The associations between inflammation and cognitive function across a broad age spectrum in cognitively unimpaired AAs with vascular risk factors require further study to ascertain pathways through which inflammation, specifically TNF $\alpha$ activity, may erode cognitive function. In addition, studies targeting modifiable vascular risk factors and effects on inflammation and cognitive function in at-risk populations are needed.

\section{ACKNOWLEDGMENTS}

American College of Physicians Abstract Day Poster Winner, American Heart Association Poster Presentation.

This work was supported by National Institutes of Health Grants U01-HL054463, R01-HL87660, HL-81331, M01 RR00585.

Conflict of Interest: The editor in chief has reviewed the conflict of interest checklist provided by the authors and has determined that the authors have no financial or any other kind of personal conflicts with this paper.

Author Contributions: Concept and design: Windham, Griswold, Mosley. Acquisition of subjects and data: Mosley, Kullo, Peyser, Bielak, Turner. Analysis and interpretation of data: Lirette, Simpson, Griswold, Windham, Mosley, Bridges. Preparation of manuscript: Windham, Simpson, Griswold, Bridges, Kullo, Peyser, Lirette, Mosley, Bielak, Turner.

Sponsor's Role: The sponsor played no role in the design, methods, subject recruitment, data collections, analysis, or preparation of paper.

\section{REFERENCES}

1. Román GC, Erkinjuntti T, Wallin A et al. Subcortical ischaemic vascular dementia. Lancet Neurol 2002;1:426-436.

2. Kalaria RN, Ballard C. Overlap between pathology of Alzheimer disease and vascular dementia. Alzheimer Dis Assoc Disord 1999;13:S115-S123.

3. Vermeer SE, Prins ND, den Heijer T et al. Silent brain infarcts and the risk of dementia and cognitive decline. N Engl J Med 2003;348:1215-1222.

4. Akiyama H, Arai T, Kondo $\mathrm{H}$ et al. Cell mediators of inflammation in the Alzheimer disease brain. Alzheimer Dis Assoc Disord 2000;14(Suppl 1): S47-S53.

5. Ridker PM, Rifai N, Rose L et al. Comparison of C-reactive protein and low-density lipoprotein cholesterol levels in the prediction of first cardiovascular events. N Engl J Med 2002;347:1557-1565.

6. Jenny NS, French B, Arnold AM et al. Long-term assessment of inflammation and healthy aging in late life: The Cardiovascular Health Study All Stars. J Gerontol A Biol Sci Med Sci 2012;67A:970-976.

7. Wright CB, Sacco RL, Rundek TR et al. Interleukin-6 is associated with cognitive function: The Northern Manhattan Study. J Stroke Cerebrovasc Dis 2006;15:34-38.

8. Engelhart MJ, Geerlings MI, Meijer J et al. Inflammatory proteins in plasma and the risk of dementia: The Rotterdam Study. Arch Neurol 2004;61:668-672.

9. Helmy AA, Abdel Naseer MM, El Shafie S et al. Role of interleukin 6 and alpha-globulins in differentiating Alzheimer and vascular dementias. Neurodegener Dis 2012;9:81-86.

10. Jefferson AL, Massaro JM, Beiser AS et al. Inflammatory markers and neuropsychological functioning: The Framingham Heart Study. Neuroepidemiology 2011;37:21-30.

11. Trollor JN, Smith E, Baune BT et al. Systemic inflammation is associated with MCI and its subtypes: The Sydney Memory and Aging Study. Dement Geriatr Cogn Disord 2010;30:569-578.

12. Buchhave $\mathrm{P}$, Zetterberg $\mathrm{H}$, Blennow $\mathrm{K}$ et al. Soluble TNF receptors are associated with $A ß$ metabolism and conversion to dementia in subjects with mild cognitive impairment. Neurobiol Aging 2010;31:1877-1884.

13. Yaffe K, Lindquist K, Penninx BW et al. Inflammatory markers and cognition in well-functioning African-American and white elders. Neurology 2003;61:76-80.

14. Laurin D, David Curb J, Masaki KH et al. Midlife C-reactive protein and risk of cognitive decline: A 31-year follow-up. Neurobiol Aging 2009;30:1724-1727.

15. Ravaglia G, Forti $P$, Maioli $F$ et al. Blood inflammatory markers and risk of dementia: The Conselice Study of Brain Aging. Neurobiol Aging 2007;28:1810-1820. 
16. Schmidt R, Schmidt H, Curb JD et al. Early inflammation and dementia: A 25-year follow-up of the Honolulu-Asia Aging Study. Ann Neurol 2002;52:168-174.

17. Marioni RE, Stewart MC, Murray GD et al. Peripheral levels of fibrinogen, C-reactive protein, and plasma viscosity predict future cognitive decline in individuals without dementia. Psychosom Med 2009;71:901-906.

18. Zuliani G, Ranzini M, Guerra G et al. Plasma cytokines profile in older subjects with late onset Alzheimer's disease or vascular dementia. J Psychiatr Res 2007;41:686-693.

19. Green RC, Cupples LA, Go R et al. Risk of dementia among white and African American relatives of patients with Alzheimer disease. JAMA 2002;287:329-336.

20. Fisher G, Hyatt TC, Hunter GR et al. Markers of inflammation and fat distribution following weight loss in African-American and white women. Obesity (Silver Spring) 2012;20:715-720.

21. Carroll JF, Fulda KG, Chiapa AL et al. Impact of race/ethnicity on the relationship between visceral fat and inflammatory biomarkers. Obesity 2009;17:1420-1427.

22. Nazmi A, Victora CG. Socioeconomic and racial/ethnic differentials of C-reactive protein levels: A systematic review of population-based studies. BMC Public Health 2007;7:212.

23. Brown MD, Feairheller DL, Thakkar S et al. Racial differences in tumor necrosis factor-alpha-induced endothelial microparticles and interleukin-6 production. Vasc Health Risk Manag 2011;7:541-550.

24. Folsom AR, Aleksic N, Catellier D et al. C-reactive protein and incident coronary heart disease in the Atherosclerosis Risk in Communities (ARIC) study. Am Heart J 2002;144:233-238.

25. Kim CX, Bailey KR, Klee GG et al. Sex and ethnic differences in 47 candidate proteomic markers of cardiovascular disease: The Mayo Clinic Proteomic Markers of Arteriosclerosis Study. PLoS One 2010;5:e9065.

26. Heald AH, Anderson SG, Ivison F et al. C-reactive protein and the insulinlike growth factor (IGF)-system in relation to risk of cardiovascular disease in different ethnic groups. Atherosclerosis 2003;170:79-86.

27. Aderka D, Engelmann H, Shemer-Avni Y et al. Variation in serum levels of the soluble TNF receptors among healthy individuals. Lymphokine Cytokine Res 1992;11:157-159.

28. Aderka D, Sorkine P, Abu-Abid S et al. Shedding kinetics of soluble tumor necrosis factor (TNF) receptors after systemic TNF leaking during isolated limb perfusion. Relevance to the pathophysiology of septic shock. J Clin Invest 1998;101:650-659.

29. Ellington AA, Kullo IJ, Bailey KR et al. Measurement and quality control issues in multiplex protein assays: A case study. Clin Chem 2009;55:10921099.

30. Folstein MF, Folstein SE, McHugh PR. 'Mini-mental state'. A practical method for grading the cognitive state of patients for the clinician. J Psychiatr Res 1975;12:189-198.

31. Morris JC, Heyman A, Mohs RC et al. The Consortium to Establish a Registry for Alzheimer's Disease (CERAD). Part I. Clinical and neuropsychological assessment of Alzheimer's disease. Neurology 1989;39:1159-1165.

32. Spreen O, Strauss E. A Compendium of Neuropsychological Tests: Administration, Norms, and Commentary. New York, NY: Oxford University Press, 1998.

33. Kaplan E, Fein D, Morris R et al. WAIS-R-NI Manual. San Antonio, TX: The Psychological Corporation, 1991.

34. Chibnik LB, Shulman JM, Leurgans SE et al. Cr1 is associated with amyloid plaque burden and age-related cognitive decline. Ann Neurol 2011;69:560-569

35. Massaro JM, D'Agostino RB Sr, Sullivan LM et al. Managing and analysing data from a large-scale study on Framingham offspring relating brain structure to cognitive function. Stat Med 2004;23:351-367.

36. Wilson RS, Beckett LA, Barnes LL et al. Individual differences in rates of change in cognitive abilities of older persons. Psychol Aging 2002;17:179-193.

37. Bohnstedt M, Fox PJ, Kohatsu ND. Correlates of Mini-Mental Status Examination scores among elderly demented patients: The influence of race-ethnicity. J Clin Epidemiol 1994;47:1381-1387.

38. Fornage M, Chiang YA, O'Meara ES et al. Biomarkers of inflammation and MRI-defined small vessel disease of the brain: The Cardiovascular Health Study. Stroke 2008;39:1952-1959.
39. Wright CB, Moon Y, Paik MC et al. Inflammatory biomarkers of vascular risk as correlates of leukoaraiosis. Stroke 2009;40:3466-3471.

40. Kuller LH, Shemanski L, Manolio $\mathrm{T}$ et al. Relationship between ApoE, MRI findings, and cognitive function in the Cardiovascular Health Study. Stroke 1998;29:388-398.

41. Knopman D, Boland LL, Mosley $\mathrm{T}$ et al. Cardiovascular risk factors and cognitive decline in middle-aged adults. Neurology 2001;56:42-48.

42. Knopman DS, Mosley TH, Catellier DJ et al. Fourteen-year longitudinal study of vascular risk factors, APOE genotype, and cognition: The ARIC MRI Study. Alzheimers Dement 2009;5:207-214.

43. Gorelick PB, Nyenhuis D, American Society of Hypertension Writing Group et al. Blood pressure and treatment of persons with hypertension as it relates to cognitive outcomes including executive function. J Am Soc Hypertens 2012;6:309-315.

44. Roger VL, Go AS, Lloyd-Jones DM et al. Heart disease and stroke statistics-2012 update: A report from the American Heart Association. Circulation 2012;125:e2-e220.

45. Dorffel Y, Latsch C, Stuhlmuller B et al. Preactivated peripheral blood monocytes in patients with essential hypertension. Hypertension 1999;34:113-117.

46. McAfoose J, Baune BT. Evidence for a cytokine model of cognitive function. Neurosci Biobehav Rev 2009;33:355-366.

47. van Himbergen TM, Beiser AS, Ai M et al. Biomarkers for insulin resistance and inflammation and the risk for all-cause dementia and Alzheimer disease: Results from the Framingham Heart Study. Arch Neurol 2012;69:594-600.

48. Tarkowski E, Blennow $\mathrm{K}$, Wallin A et al. Intracerebral production of tumor necrosis factor-alpha, a local neuroprotective agent, in Alzheimer disease and vascular dementia. J Clin Immunol 1999;19:223-230.

49. Jordanova V, Stewart R, Davies E et al. Markers of inflammation and cognitive decline in an African-Caribbean population. Int J Geriatr Psychiatry 2007;22:966-973.

50. van den Biggelaar AHJ, Gussekloo J, de Craen AJM et al. Inflammation and interleukin-1 signaling network contribute to depressive symptoms but not cognitive decline in old age. Exp Gerontol 2007;42:693-701.

\section{SUPPORTING INFORMATION}

Additional Supporting Information may be found in the online version of this article:

Figure S1. Associations Between standardized inflammatory markers and cognitive domains, adjusted for age, sex, and education and accounting for familial clustering. Regression coefficients with lower and upper confidence limits are shown. Black lines indicate statistically significant results. sTNFR, soluble tumor necrosis factor receptor; CRP, high sensitivity C-reactive protein; IL6, interleukin 6; MMSE, Mini-Mental State Examination; PS, Processing Speed; executive function, Executive Function.

Tables S1. Relationships Between Cognitive Domains and Inflammatory Markers in European and African Americans. Sensitivity to Missing Cognitive Outcomes Comparing Generalized Estimating Equations (GEE) and Weighted GEE (wGEE).

Please note: Wiley-Blackwell is not responsible for the content, accuracy, errors, or functionality of any supporting materials supplied by the authors. Any queries (other than missing material) should be directed to the corresponding author for the article. 